\title{
Attention Biases Competition for Visual Representation via Dissociable Influences from Frontal and Parietal Cortex
}

\author{
Andrew D. Sheldon ${ }^{1 *}$, Elyana Saad ${ }^{2}$, Muhammet I. Sahan ${ }^{3}$, Emma E. Meyering ${ }^{1}$, \\ Michael J. Starrett ${ }^{4}$, Joshua J. LaRocque ${ }^{5}$, Nathan S. Rose ${ }^{6}$, and Bradley R. Postle ${ }^{\text {* }}$
}

\begin{abstract}
What mechanisms underlie the prioritization of neural representations of visually perceived information to guide behavior? We assessed the dynamics whereby attention biases competition for representation of visual stimuli by enhancing representations of relevant information and suppressing the irrelevant. Multivariate pattern analysis (MVPA) classifiers were trained to discriminate patterns of fMRI activity associated with each of three stimuli, within several predefined ROIs. Participants performed a changedetection task wherein two of three presented items flashed at $1 \mathrm{~Hz}$, one to each side of central fixation. Both flashing stimuli would unpredictably change state, but participants covertly counted the number of changes only for the cued item. In the ventral occipito-temporal ROI, MVPA evidence (a proxy for representational fidelity) was dynamically enhanced for attended stimuli
\end{abstract}

\section{INTRODUCTION}

In human neuroimaging, information-based multivariate approaches to data analysis have revealed a wealth of insights into the mechanistic implementation of populationlevel neural representation (Huth et al., 2016; Sprague, Saproo, \& Serences, 2015; van Bergen, Ma, Pratte, \& Jehee, 2015; Haxby et al., 2001). In studies of selective attention, for example, multivariate analyses applied to fMRI data have demonstrated that, in occipito-temporal regions responsible for visual object recognition, attention has the effect of biasing high-dimensional patterns of activity toward the pattern observed when the cued stimulus is presented alone, and away from the pattern observed when the uncued pattern is presented alone (Cohen \& Tong, 2015; Reddy, Kanwisher, \& VanRullen, 2009). This is consistent with the idea that the mechanism of biased competition governs competition for representation in the human visual system in a manner similar to how it has been characterized in the monkey (Desimone \& Duncan, 1995).

\footnotetext{
${ }^{1}$ University of Wisconsin-Madison, ${ }^{2}$ Harvard Medical School, ${ }^{3}$ Ghent University, ${ }^{4}$ University of California, Davis, ${ }^{5}$ New York University, ${ }^{6}$ University of Notre Dame

*These authors contributed equally to this work.
}

and suppressed for unattended stimuli, consistent with a mechanism of biased competition between stimulus representations. Frontal and parietal ROIs displayed a qualitatively distinct, more "source-like" profile, wherein MVPA evidence for only the attended stimulus could be observed above baseline levels. To assess how attentional modulation of ventral occipito-temporal representations might relate to signals originating in the frontal and/or parietal ROIs, we analyzed informational connectivity (IC), which indexes time-varying covariation between regional levels of MVPA evidence. Parietal-posterior IC was elevated during the task, but did not differ for cued versus uncued items. Frontal-posterior IC, in contrast, was sensitive to an item's priority status. Thus, although regions of frontal and parietal cortex act as sources of top-down attentional control, their precise functions likely differ.
As with many models of attention, biased competition models distinguish between the site where attention has its effects and the source of the top-down signals that implement the task-dependent prioritization of a subset of the information currently being processed in the visual system. In some theoretical accounts, this source of attentional control is isomorphic with the search template, held in working memory, that corresponds to the trial-by-trial focus of behavioral priority (Van der Stigchel \& Olivers, 2019; Reinhart \& Woodman, 2014; Hamker, 2005). Empirically, the source of the endogenous control of attention has been associated with frontoparietal circuits whose activity varies with demands for attentional control (Corbetta \& Shulman, 2002), for which trial-by-trial fluctuation in (univariate) signal intensity covaries with multivariate pattern analysis (MVPA) decoding of stimulus information in ventral occipito-temporal (VOT) cortex (Nelissen, Stokes, Nobre, \& Rushworth, 2013), and which acts as a driver of attention-related shifts in oscillatory synchrony with domain-selective regions of VOT (Baldauf \& Desimone, 2014).

Recently, the idea of a functional distinction between VOT regions as sites of the effects of endogenous attentional control, versus frontoparietal circuits as sources of this control, has been called into question based on 
multivariate evidence for stimulus-specific information in putatively control-related frontoparietal systems. Ester, Sutterer, Serences, and Awh (2016) acquired fMRI data while participants attended to either the luminance or the orientation of a grating stimulus, and determined with multivariate inverted encoding modeling that stimulus orientation was represented in many areas of cortex, not only in VOT regions but also in intraparietal sulcus (IPS) and in frontal cortex, including in superior and inferior precentral sulcus (iPCS). Furthermore, and importantly for the current study, in some of these regions, including IPS and iPCS, these representations of stimulus information were modulated by attention, with inverted encoding modeling indicating higher-amplitude representations during attend-orientation blocks of trials.

These findings from Ester et al. (2016) are emblematic of a broader trend in neuroimaging studies of visual cognition, which is that powerful multivariate analyses applied to these data sets are finding evidence for stimulus-specific information in regions classically associated with more abstract and/or categorical representation of information, as well as with the representation and implementation of rules that determine how behavior will be guided by visual representations. Many recent studies of visual working memory, for example, have reported the delay-period representation of trial-specific analog features of sample stimuli in IPS and in frontal cortex, as well as in VOT cortex (e.g., Cai, Sheldon, Yu, \& Postle, 2019; Christophel, Iamshchinina, Yan, Allefeld, \& Haynes, 2018; Gosseries et al., 2018; Bettencourt \& Xu, 2016; Ester et al., 2016; Christophel \& Haynes, 2014; Christophel, Hebart, \& Haynes, 2012). Determining, in such instances, whether multiple regions are supporting the same function(s), or whether they are supporting distinct functions that are nonetheless all being carried out on the same information, is an important goal for cognitive neuroscience research (cf. Christophel, Klink, Spitzer, Roelfsema, \& Haynes, 2017).

Returning to models of feature-based selective attention, the focus of this paper, Ester et al. (2016) suggest that their findings "challenge models that posit a strict segregation between sources and sites of attentional control on the basis of representational properties by demonstrating that simple feature values are encoded by cortical regions throughout the visual processing hierarchy, and that representations in many of these areas are modulated by attention" (p. 8188). This interpretation merits careful scrutiny, because its adoption would require the rejection of a decades-old core assumption about the control of attention. Indeed, we do not believe that the results from the Ester et al. (2016) study, alone, represent a strong challenge to the source-versus-site distinction, because of two limitations of its experimental design: first, participants in the study were presented with only one item per trial; second, the to-be-attended feature was blocked. The first factor is important because a cardinal property of attention is its role in resolving the competition for representation between two or more objects or features in the visual scene. This is reflected, of course, in the very name of the biased competition model that is of central interest for this study and is also seen empirically from several perspectives. Behaviorally, displays that trigger an exogenous capture of attention (i.e., pop-out) do not place demands on endogenously controlled selection and, therefore, on the mechanisms that correspond to the idea of a source of attentional control. Mechanistically, in experiments where subthreshold microstimulation of neurons in the FEF is believed to act like an experimenter-controlled source of top-down attentional signals, attention-like enhancements of the visually driven response of V4 neurons are only seen when a distractor is also present in the display, elsewhere in the visual field (Moore \& Armstrong, 2003). Although one could argue that the stimuli used by Ester et al. (2016) required selection of one from among two features (i.e., they were compound stimuli), competition between these two features would be expected to be minimized by the second limiting factor in this study's design, the fact that the to-be-attended feature was the same on every trial within a block. This is because blocking of search targets quickly minimizes the need for an active representation of the search template and, therefore, quickly minimizes the need for top-down control (Reinhart \& Woodman, 2014; Carlisle, Arita, Pardo, \& Woodman, 2011). Finally, the data from Ester et al. (2016) do not rule out the alternative interpretation that the attention-related modulation of stimulus representations in frontal and parietal regions may have reflected changes in the state of circuits acting as a source of top-down control. By analogy, when the focus of spatial attention is shifted from one area of the visual field to another, activity decreases both in FEF neurons with motor fields in the first area and $\mathrm{V} 4$ neurons with receptive fields in the first area (Moore \& Zirnsak, 2017), but it does not follow from this that these two groups of neurons are supporting the same function. Relatedly, one might also expect representations implementing the control of feature-based attention to change as a function of priority (cf. Bichot, Heard, DeGennaro, \& Desimone, 2015).

The present experiment was designed to implement a direct assessment of biased competition in human visual attention by presenting two flashing, complex stimuli, one in each visual field, and instructing participants to attend to one or the other with two sequential cues. The category of stimuli in this two-item "search array" varied on a trial-by-trial basis, and cuing was unpredictable. We used MVPA of fMRI activity as a proxy for the strength of the representation of task-related information. Our results replicated previous findings from the monkey that the multivariate decoding of object information in the ventral visual stream is sensitive to the current state of priority (Zhang et al., 2011), consistent with an effect of attention of biasing the strength of population-level stimulus representations in visual cortex. Furthermore, although stimulus information could also be decoded in PFC and parietal regions, the patterns of sensitivity to attention in these 
regions were markedly different than those in VOT cortex, consistent with the idea that stimulus information in PFC and parietal cortex may support different functions than those in VOT cortex. Finally, using a multivariate metric of "informational connectivity" (IC; Coutanche \& ThompsonSchill, 2014), we find evidence for a specific role for PFC in the suppression of unprioritized information.

\section{METHODS}

\section{Participants}

Ten healthy participants ( 6 women, $M_{\text {age }}=22.4$ years old; $S D=5.08$ years) with normal or corrected-to-normal vision received monetary compensation for participating in the study. All participants were screened to rule out any neurological and psychiatric disorders, as well as incompatibility with MRI, and all provided written informed consent. The study was approved by the UW-Madison institutional review board.

\section{Behavioral Tasks}

Two tasks were performed during fMRI scanning, a oneitem change-detection task (used to train MVPA classifiers) and a two-item cued change-detection task (the principal experimental task). Both tasks used three pairs of stimuli taken from the image set of Brady, Konkle, Alvarez, and Oliva (2008): a woman's face, the two tokens portraying subtly different expressions; a doughnut, each token with a different sized bight taken out of it; and an abacus, the two tokens with the beads arranged differently. Each image was cropped into a square aperture subtending approximately $3^{\circ}$ of visual angle along all edges. Screen background was gray, and fixation cross was white (Figure 1A).

Each run of the one-item task consisted of sixteen 18-sec mini-blocks, during 12 of which images from one of the stimulus categories were presented centrally, flickering with a cycle of $750 \mathrm{msec}$ on/250 msec off (four mini-blocks for each of the image categories, the remaining four miniblocks presenting a fixation cross that did not flicker.) Each of the 12 stimulus-presenting mini-blocks began with $1 \mathrm{sec}$ of fixation, followed by $15 \mathrm{sec}$ of flicker. The first image to appear was randomly selected from the pair, and on the remaining 14 cycles, the identity of the image could change to the other from that category, unpredictably, on zero, one, or two occasions. Participants were instructed to count the number of "state changes" that the target underwent during the mini-block, and to report that number with a button press during the final 2 sec of the mini-block. There were no intervals separating mini-blocks. Each participant performed six runs of the one-item task, the order of mini-blocks randomized for each run.

The two-item task was administered in multistep trials, each consisting of an initial stimulus presentation and delay, followed by two change-detection epochs. Each trial began with the 500-msec display of that trial's two potential search targets, each from a different one of the three

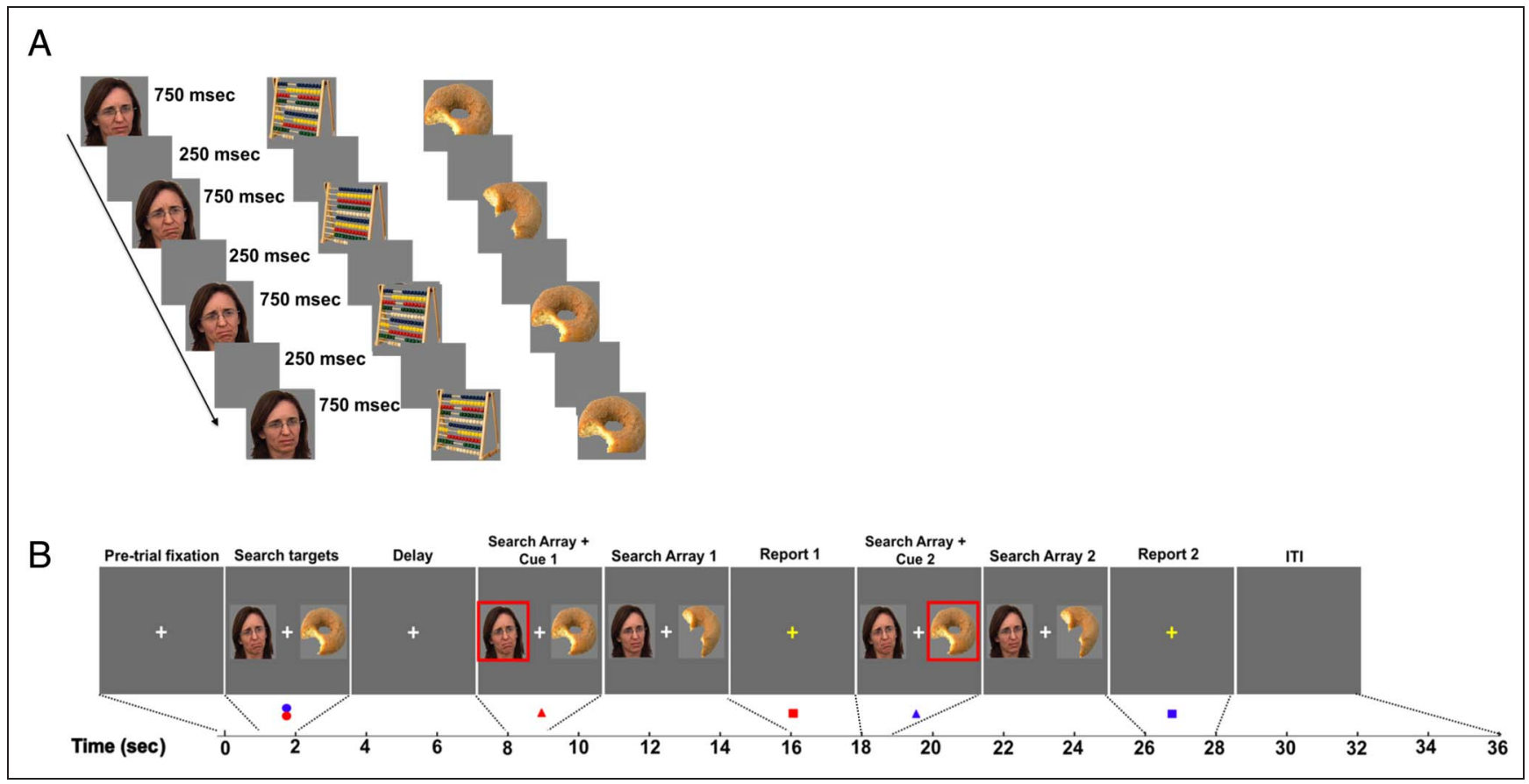

Figure 1. Stimuli and tasks. (A) The three stimulus categories, each shown as four cycles from a mini-block of the one-item task. The face is shown changing state between the first and second image and between the third and fourth; the abacus is shown changing state between the first and second and between the second and third; the doughnut between the first and second and between the second and third. (B) Timeline of the two-item task (the principal experimental task), illustrating a switch trial. 
possible categories, each centered $5^{\circ}$ of visual angle along the horizontal meridian to the left or right of fixation. The offset of these images was followed by a 7-sec blank delay, after which the two images reappeared, each in the same location, this time one of them cued by being outlined by a red box (500-msec duration) indicating that it was the item whose changes were to be tracked across the ensuing 7.5-sec change-detection epoch (the "search array"). During this change-detection epoch, the two images each flickered at 1-Hz (750 msec on, $250 \mathrm{msec}$ off), each changing state unpredictably, independently, 0,1 , and 2 times. At the end of the epoch, the flickering display was replaced by a prompt to report the number of changes in the cued image $(2 \mathrm{sec})$, followed by a second search array featuring the same elements and timing as the first, the second cue having cued, unpredictably, the previously cued item on half the trials ("Stay" cues), and the previously uncued item on half the trials ("Switch" cues). The intertrial interval was $10 \mathrm{sec}$. Each block of the two-item task included 12 trials. (Figure 1B).

Each participant performed six blocks of the one-item task and four blocks of the two-item task, half of the participants performing the one-item task first, the other half performing the two-item task first.

\section{fMRI Scanning Session}

\section{Data Acquisition}

Whole-brain images were acquired with the 3 T MRI scanner (Discovery MR750; GE Healthcare) at the Lane Neuroimaging Laboratory at the University of Wisconsin-Madison. Highresolution T1-weighted images were acquired for all participants with a fast spoiled gradient echo sequence (repetition time $[\mathrm{TR}]=8.132 \mathrm{msec}$, echo time $=3.18 \mathrm{msec}$, $12^{\circ}$ flip angle, 156 axial slices, $256 \times 256$ in. plane, $1.0 \mathrm{~mm}$ isotropic). BOLD-sensitive data were acquired using a gradient-echo, echoplanar sequence $(\mathrm{TR}=2 \mathrm{~s}$, echo time $=$ $25 \mathrm{msec}$ ) within a $64 \times 64$ matrix (39 sagittal slices, $3.5 \mathrm{~mm}$ isotropic).

\section{Eye-Tracking}

To ensure that participants maintained fixation during task performance, we recorded eye position in the scanner using EOG monitored with Brain Vision software and recording equipment (BrainVision recording suite, Brain Products, www.brainproducts.com/index.php). Participants were excluded if saccades were determined to have been made during the search array portions of $>10 \%$ of trials (as determined by an observed deflection on EOG of $>25 \mu \mathrm{V}$, consistent with a saccade of $1^{\circ}-2^{\circ}$ of visual angle). No participants met this threshold, and so all were included, including trials where saccades were made, as they constituted a small $(<10 \%)$ portion of trials overall, and to avoid potentially disrupting the relative balance of stimulus types in the data set.

\section{fMRI Data Analysis}

Preprocessing, univariate general linear modeling, and ROI definition were performed using the Analysis of Functional NeuroImages software package (afni.nimh.nih.gov).

\section{Preprocessing}

We excluded the first three volumes of each run to account for EPI-onset field inhomogeneity. All volumes were spatially realigned to the final volume of the final functional run using rigid-body realignment. The processing pipeline included slice time correction, detrending, conversion to percent signal change, and spatial smoothing with a 4-mm FWHM Gaussian kernel.

\section{Univariate Analyses}

To generate functionally defined ROIs, we modeled the fMRI data from the one-item task with a general linear model, including regressors-of-interest that were 18-sec boxcars spanning each mini-block, labeled by stimulus category, and convolved with a canonical hemodynamic response function, as well as nuisance regressors to remove effects of head motion and low-frequency drift.

\section{Generation of ROIs}

To generate ROIs for the MVPA, we first generated anatomical masks using the Talraich anatomical atlas (sscc.nimh .nih.gov/afni/doc/misc/afni_ttatlas/), selecting from the atlas the gyri wanted for each brain region, then warping these into each participant's native space. A VOT anatomical ROI comprised lingual gyrus, fusiform gyrus, inferior occipital gyrus, inferior temporal gyrus, middle occipital gyrus, and superior occipital gyrus; a parietal ROI comprised posterior cingulate, orbital gyrus, precuneus, inferior parietal lobule, and superior parietal lobule, and a frontal ROI comprised the inferior frontal, middle frontal, superior gyrus, medial frontal, and precentral gyri. Next, functional ROIs were created for each of these regions by masking the results from the contrast [(face + doughnut + abacus) fixation] with each of the three ROIs. For bilateral ROIs, we selected within each anatomical ROI the 400 voxels with the highest $F$ statistic. For unilateral ROIs, we first divided each the three regional ROIs by hemisphere and then selected within each hemisphere-specific anatomical ROI the 200 voxels with the highest $F$ statistic. We have used this approach to ROI generation in several prior studies in our laboratory (LaRocque, Riggall, Emrich, \& Postle, 2017; Rose et al., 2016; Emrich, Riggall, Larocque, \& Postle, 2013), and it has the advantage of accounting for individual differences in anatomical patterns of task-relevant neural activity.

\section{Pattern Classification Analyses}

MVPA was implemented using L2-regularized logistic regression with a lambda penalty term of 25 , performed 
using the Princeton Multi-Voxel Pattern Analysis toolbox (www.pni.princeton.edu/mvpa/) and custom scripts in MATLAB (The MathWorks). Before MVPA, all neural data were $z$-scored across trials, within runs.

Classifiers were trained on data from the middle-to-late portions of each mini-block (TRs 4-8) of the one-item task, to learn to discriminate neural patterns associated with the three stimulus categories. To assess classifier sensitivity, we used k-fold cross-validation $(k=6)$, training a classifier 6 times, leaving out a different run each time, and then averaging across folds. Statistical significance of classifier accuracy was computed by performing a one-sample, onetailed $t$ test comparing accuracy to chance performance (33\%). Once validated, we applied classifiers trained on data from Experiment 1 to all time points in Experiment 2, sorting the evidence outputs by attentional state.

The classifiers were trained on data from the one-item task and were then applied to the data from each time point of the two-item task. First, a measure of pattern similarity was computed between the voxel patterns of each time point in the testing set and the learned pattern for each category derived from training on the one-item data. Using logistic regression, each category's pattern similarity score was then converted into a value ranging between 0 and 1 , analogous to an estimate of probability that the observed testing pattern was generated by that category. This measure of classifier evidence for each of the three stimulus categories at each time point in the trial was sorted according to whether that category was initially cued, initially uncued, or absent on each trial and then averaged together, generating a trial-averaged time course. Critically, classifier evidence for the absent category would serve as an empirical estimate of baseline. Because the category that was cued on the second half of the trial was different after switch cues versus stay cues, these trial types were averaged, and are presented, separately.

\section{Ipsilateral versus Contralateral Region Analyses}

We were also interested in assessing the effects of prioritization as a function of whether the cued stimulus appeared contra- or ipsilateral to a given brain region. To do this, classifiers were trained as above to distinguish stimulus category using the unilateral ROI data (see Generation of ROIs section) generated during the one-item task, in which all stimuli were presented foveally, and then tested this classifier on data from the same ROI with data from the two-item task, in which stimuli were presented peripherally (one in each visual hemifield). Data from trials were then concatenated for each region according to whether that region was initially contralateral to the initially cued stimulus or ipsilateral to it to generate the "contralateral" and "ipsilateral" plots, respectively.

\section{Bayesian Statistics}

Because several studies from our group have found that MVPA evidence for stimulus representation often drops to baseline levels when one of two items held in working memory is not prioritized by a priority cue (LaRocque et al., 2017; Rose et al., 2016; Emrich et al., 2013), we applied Bayesian statistics to quantify the probability that evidence for stimulus representations was different between categories, including whether uncued information differed from baseline. In this experiment, MVPA evidence for an absent stimulus can be understood as the empirical baseline of our method. Therefore, epochs during which MVPA evidence for a trial-relevant stimulus exceeds MVPA evidence for the absent stimulus are interpreted as evidence of an active neural representation of that stimulus, and the magnitude of that difference a proxy for the strength of the neural representation (Emrich et al., 2013). The Bayes factor is the ratio of the likelihood of the alternative hypothesis compared with the null hypothesis, each of which incorporates an estimate of the prior probability of that hypothesis. Therefore, to calculate the Bayes factor, it is necessary to provide a prior probability distribution for the alternative hypothesis, for which we used a previously published and validated calculator (Dienes, 2014). In defining this prior distribution, a minimum plausible value was set at a small difference above 0 (0.01), and a maximum plausible value at the maximum evidence achieved in the one-item task ( 0.7 , the difference in evidence between the correct and the two incorrect stimulus types on each mini-block). In addition, to avoid potentially biasing the resulting calculations, the simplest possible prior was chosen for the alternative hypothesis, a uniform distribution extending from zero difference to the maximum plausible difference. Alternatives such as a halfGaussian distribution centered at the maximum plausible value or a Gaussian centered at the midpoint were also considered, but these were ultimately rejected given a lack of sufficient evidence to presuppose any one distribution over the other. Regardless, Bayes factors calculated using those prior distributions agreed substantively with those of the uniform distribution.

In addition, because the trial sections of greatest interest were the 7-sec delay period after the presentation of that trial's potential search targets and the two 7.5-sec searcharray epochs, evidences from the final three TRs of each of those segments were averaged together (after accounting for hemodynamic lag) and used for the Bayes factor (BF) calculations. Finally, because Bayes factors can be thought of as the ratio of evidence for the alternative hypothesis compared to the null hypothesis, multiple Bayes factors calculated for the same ratio of hypotheses can be combined to express a total probability through simple multiplication (Dienes, 2014).

\section{Comparison of Region, Search Array Period, and Stay/Switch Condition Differences}

To compare across ROIs, stay versus switch trials, and the different search array epochs, we conducted two repeatedmeasures ANOVAs that included each of these variables as 
factors, along with a variable of cue status (initially cued, initially uncued, absent). One of these ANOVAs was carried out on data from the bilateral ROIs and one on data from the unilateral ROIs. All variables were within participant. Because of the very clear pattern in the results (and to more directly and explicitly operationalize the idea of a general "anterior versus posterior" distinction between "source-like" versus "site-like" functions, respectively), both ANOVAs were carried out by simply labeling the MVPA data for frontal and parietal sources as the same ROI in the regression. For both ANOVAs, MVPA evidences from all participants were regressed onto these variables using the fitrm function in MATLAB, and statistics were computed with the ranova function.

\section{IC}

The estimates of classifier evidence from the Search Array 1 epoch were also used to compute a measure of IC (Coutanche \& Thompson-Schill, 2014) between ROIs. IC is analogous to functional connectivity, but rather than assessing patterns of covariation in raw neuroimaging signal, it correlates the time course of the classifier evidence. Importantly, IC assesses whether two areas are sharing information of interest, whereas functional connectivity would be unable to discriminate this scenario from one in which activity between two areas is correlated, but for reasons that are unrelated to the information of interest. In other words, IC enables correlated signals to be distinguished from correlated noise. An additional advantage of IC for our design is that, because it is specific to a category of information, it can be computed separately for attended information and for unattended information measured during the same trials.

IC analyses were carried out using a modified version of the freely available online toolbox for MATLAB (lrdc.pitt .edu/coutanche/informationalconnectivity). Classifier evidences for each stimulus category (Face, Doughnut, Abacus; relabeled to Attended, Unattended, Absent) were obtained using the procedure outlined previously (training on Experiment 1 and testing on Experiment 2) for each area. This was done separately for each time point for each trial, giving rise to an evidence time course in each area for each stimulus category (attended, unattended, and absent) that was 144 time points long (48 trials $\times 3$ time points from the Search Array 1 epoch). Because evidence for the absent category represents an empirical baseline by which to gauge classifier noise, as a normalization step, the absent evidence time course was then subtracted from both the attended and unattended time courses to yield an attended discriminability time course and unattended discriminability time course, respectively. Attended discriminability time courses in one ROI were then correlated (using a nonparametric Spearman's rank method) with attended discriminability time courses in a different ROI to generate a measure of IC between those ROIs. The same was then done for unattended discriminability time courses.
To calculate significance, IC values across participants were bootstrapped to generate a distribution of possible means given the hypothesis that our sample distribution represented an accurate sampling of the underlying population. More specifically, 10,000 separate bootstrapped samples were created, drawing with replacement and equal probability 10 IC values from our pool of 10 participants to generate a new "sample" of 10 participants, some of which were likely to be repeats. For each of these bootstrapped samples, an average IC was calculated, generating a distribution of 10,000 different IC averages. The $p$ values reported here are the percentage of bootstrapped sample averages that crossed 0 , effectively generating the confidence interval. For example, if $>95 \%$ of the bootstrapped averages were positive and $<5 \%$ of the distribution was negative, then it could be said that a 95\% confidence interval did not include 0 , or the null. A similar analysis was performed for the direct comparison between attended and unattended IC for each ROI pair. First, the unattended IC value was subtracted from the attended IC value to generate an IC difference score for each ROI pair. To calculate the significance of this score, these IC difference scores were then bootstrapped as above. The average value and $p$ value of the bootstrapped samples are reported here. For completeness, BFs were also computed for each comparison and are reported along with the $p$ values generated via the bootstrap analysis. However, unlike for the MVPA evidences listed above, a more refined expected range of values for the alternative hypothesis was unable to be determined, and so a uniform prior extending from -1 to 1 was used.

\section{RESULTS}

\section{Behavior}

Participants attained a high degree of accuracy on both the one-item task $\left(86.7 \% ; S D=4.24, \mathrm{BF}=1.36 \times 10^{45}\right)$ and the two-item task $\left(84.8 \% ; S D=4.20 ; \mathrm{BF}=1.47 \times 10^{43}\right)$. Across the tasks, accuracies were similar for the three categories (doughnut 83.4\%; $S D=5.71 ; \mathrm{BF}=9.89 \times 10^{21}$ ), face $\left(83.44 \% ; S D=5.62 ; \mathrm{BF}=5.68 \times 10^{22}\right)$, and abacus $\left(87.5 \% ; S D=7.48 ; \mathrm{BF}=3.61 \times 10^{14}\right)$. Bayes factors here represent the comparisons between the value observed and chance (25\%, because participants could select from the attended object changing $0,1,2$, or 3 times in their response).

\section{fMRI Classifier Training}

Across all participants, leave-one-run-out cross-validation analyses of data from the one-item task demonstrated robust levels of classification in all ROIs, with classifier performance highest for VOT, followed by parietal, followed by frontal cortex (Figure 2). 
Figure 2. ROIs and decoding from the one-item task. (A) Several views of inflated brains showing the 400-voxel bilateral ROIs in each of three brain regions, for a representative participant. (B) Group-level decoding accuracies, by ROI.

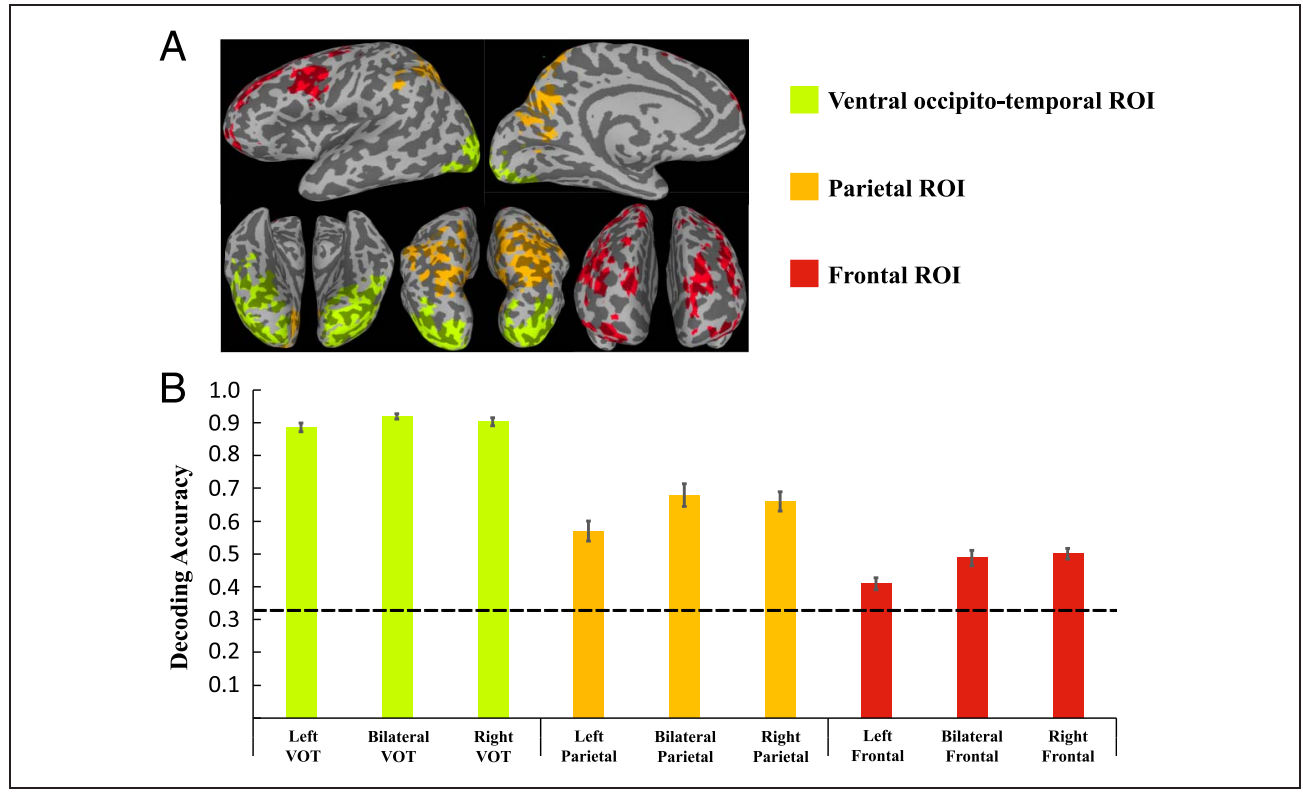

\section{Attentional Prioritization during Change Detection}

\section{Effects within Bilateral ROIs}

During the initial delay period of each trial, in all three ROIs, classifier evidence for both presented stimuli (Figure 3, red and blue traces) was elevated above evidence for the third, which was absent from that trial and thus served as an empirical baseline evidence (Figure 3, black trace; BFs, collapsed across stay and switch trials: VOT cued $>$ absent $=225$, VOT uncued $>$ absent $=$ $2.25 \times 10^{10} ;$ parietal cued $>$ absent $=11.6$, parietal uncued $>$ absent $=2.99 \times 10^{7}$; and frontal uncued $>$ absent $=153$ ). The only exception was the red trace for the initial delay in frontal cortex, which appears elevated above baseline but whose BF favors no difference (frontal uncued $>$ absent $\mathrm{BF}=.15$ ).

In VOT cortex (Figure 3A), the onset of Cue 1 and Search Array 1 had three effects on indices of neural representation. First, MVPA evidence for the absent (baseline) category dropped dramatically over the next $4-6 \mathrm{sec}$, relative to the preceding delay period (precue absent $>$ post-cue1 absent Stay BF $=6.67 \times 10^{4}$; Switch BF $\left.=275\right)$. Second,
Figure 3. Time course of classifier evidence in the two-item task, in the bilateral ROIs. Ribbons display classifier evidence, interpolated across the 19 time points in each trial, with the center representing the mean value and the edges denoting \pm 1 SEM. Colored symbols at bottom of plots indicate onsets of search targets, of cues, and of report epochs (see Figure 1).

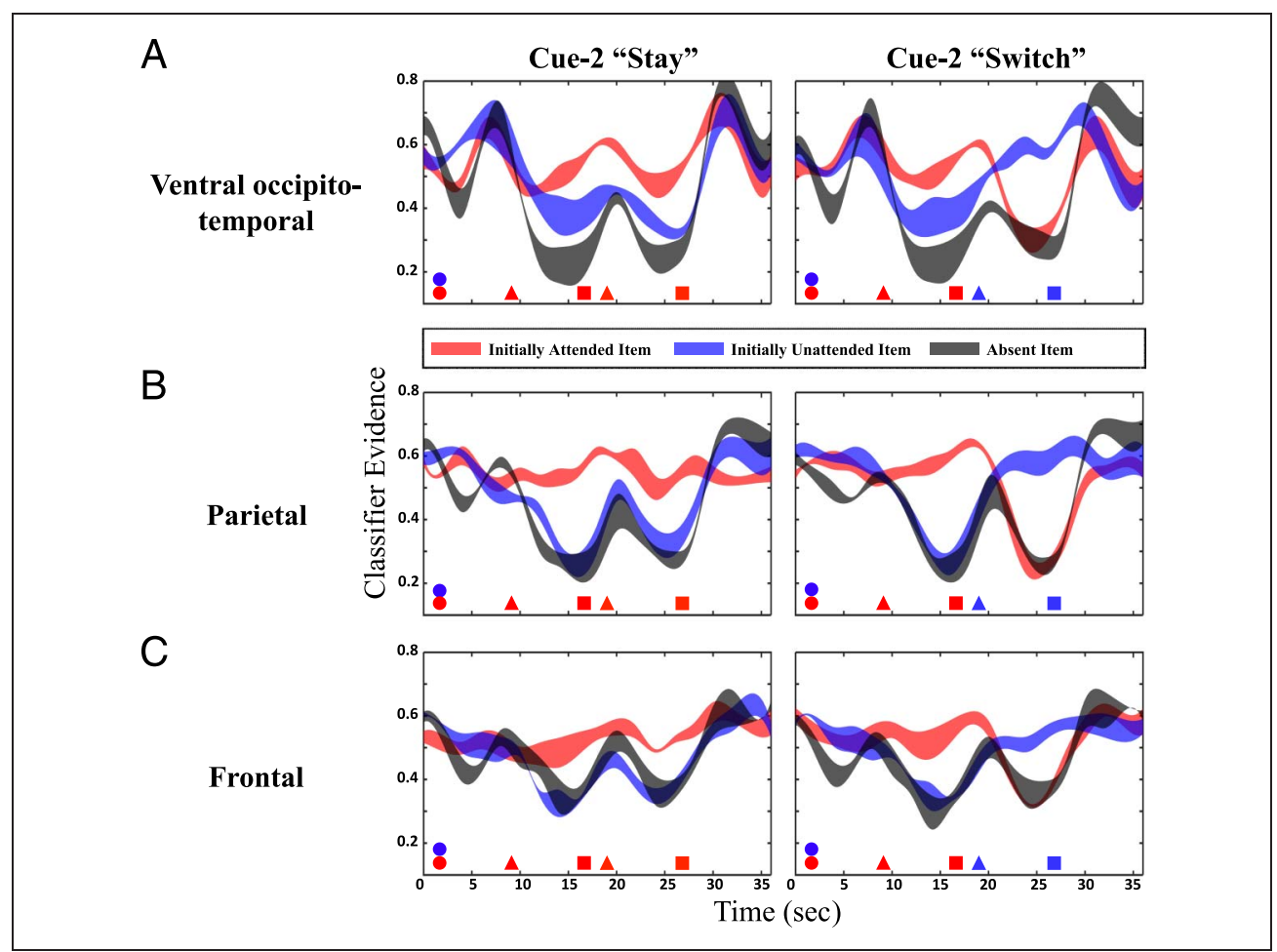


MVPA evidence for the uncued stimulus also dropped, although it remained elevated above baseline (i.e., above the absent category: uncued $>$ absent Stay $\mathrm{BF}=2.98 \times$ $10^{11}$; Switch BF $\left.=2.52 \times 10^{4}\right)$. Third, MVPA evidence for the cued stimulus diverged from that of the uncued stimulus and remained elevated over uncued for the remainder of Search Array 1 (cued $>$ uncued Stay BF $=2.8 \times 10^{4}$; Switch $\left.\mathrm{BF}=1.24 \times 10^{8}\right)$. After Stay cues, the pattern from Search Array 1 persisted across Search Array 2 (cued $>$ absent $\mathrm{BF}=2.33 \times 10^{11}$; uncued $>$ absent $\mathrm{BF}=3.49$; cued $>$ uncued $\mathrm{BF}=8.79 \times 10^{7}$ ). After Switch cues, MVPA evidence for the two stimuli crossed over, with MVPA evidence for the initially uncued/unprioritized (but now cued/prioritized) stimulus now becoming elevated above baseline to a degree comparable that of the initially cued item during Search Array 1 (now-cued $>$ absent BF $=$ $5.65 \times 10^{17}$; Initially Cued Array $1>$ Now-Cued Array 2 $\mathrm{BF}=0.0432$ ) and MVPA evidence for the initially cued/ prioritized (but now uncued) stimulus dropping to baseline levels for much of Search Array 2 (now-uncued $>$ absent $\mathrm{BF}=0.04$ ). To summarize in terms of the cognitive and neural constructs that our experiment was designed to examine, the effect of Cue 1 on the processing of the search array was to 1) "tune" the visual system in a trialspecific manner (inferred from the drop in MVPA evidence for the absent stimulus), 2) prioritize the neural representation of the cued stimulus over that of the uncued stimulus, and 3) sustain a weakened representation of the uncued item during Search Array 1. (The status of the neural representation of the uncued item during Search Array 2 was less clear and will be taken up again in the Discussion.)

Unlike VOT cortex, the effects of attentional selection on stimulus representation in the parietal and frontal ROIs were more absolute, in that the neural representation of the uncued stimulus dropped to, and subsequently remained at, baseline throughout the duration of each search array. In parietal cortex (Figure 3B), the onset of Cue 1 and
Search Array 1 triggered a drop in MVPA evidence for both the absent category and the uncued stimulus, with MVPA evidence for the uncued not differing from baseline during Search Array 1 (uncued $>$ absent Stay BF $=0.1$; Switch $\mathrm{BF}=0.09)$. Also during Search Array 1, MVPA evidence for the cued stimulus diverged from that of the uncued stimulus and remained elevated over both the uncued stimulus and the absent category for the remainder of Search Array 1 (cued $>$ uncued Stay BF $=517$; cued $>$ uncued Switch BF $=1.12 \times 10^{3}$; cued $>$ absent Stay BF $=$ $8.3 \times 10^{4}$; cued $>$ absent Switch BF $=2.13 \times 10^{6}$ ). After Stay cues, the pattern from Search Array 1 persisted across Search Array 2 (cued $>$ uncued $\mathrm{BF}=889$; cued $>$ absent $\mathrm{BF}=6.08 \times 10^{7} ;$ uncued $>$ absent $\left.\mathrm{BF}=0.16\right)$. After Switch cues, MVPA evidence for the two stimuli crossed over, with MVPA evidence for the initially unprioritized (but now prioritized) stimulus taking on a value comparable that of the initially cued item during Search Array 1 (now-cued $>$ absent $\mathrm{BF}=7.72 \times 10^{13}$; initially cued Search Array $1>$ now-cued search array $2 \mathrm{BF}=0.0809$ ), and MVPA evidence for the initially prioritized (but now unprioritized) stimulus dropping to baseline levels for much of Search Array 2 (now-uncued $>$ absent $\mathrm{BF}=0.03$ ).

In frontal cortex (Figure 3C), the patterns were qualitatively the same as for parietal cortex. Onset of Cue 1 and Search Array 1 triggered a drop in MVPA evidence for both the absent category and the uncued stimulus, with MVPA evidence for the uncued not differing from baseline (absent category) during Search Array 1 (uncued $>$ absent Stay BF $=0.1$; Switch BF $=0.09$ ). Also during Search Array 1, MVPA evidence for the cued stimulus diverged from that of the uncued stimulus and remained elevated over uncued stimulus and the absent category for the remainder of Search Array 1 (cued $>$ uncued Stay BF $=$ 0.48 ; cued $>$ uncued Switch $\mathrm{BF}=0.34$; cued $>$ absent Stay BF $=2.74 \times 10^{5}$; cued $>$ absent Switch BF $=1.68 \times$ $10^{5}$ ). After Stay cues, a qualitatively similar pattern from
Figure 4. Time course of classifier evidence in the two-item task, in unilateral VOT ROIs. (A) The hemisphere contralateral to the initially attended item. (B) The hemisphere ipsilateral to the initially attended item. All graphical conventions are the same as Figure 3.

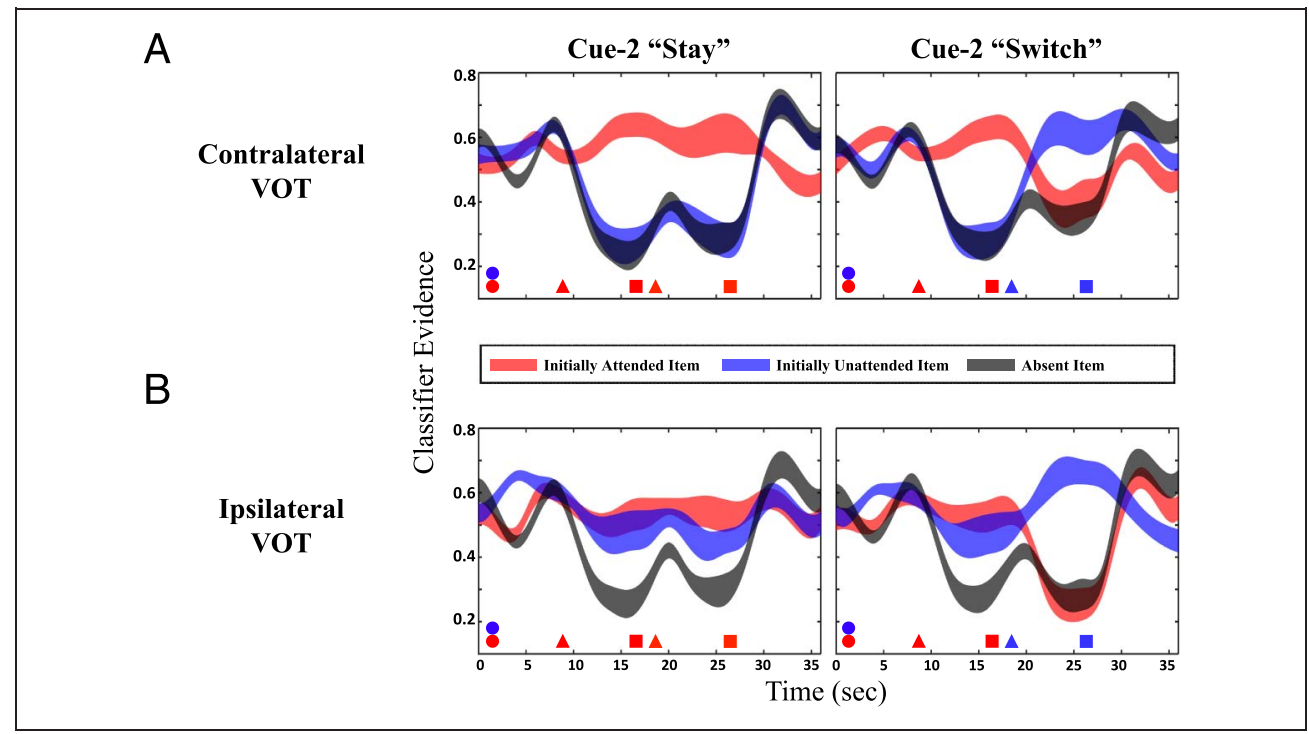


Table 1. Bayes Factor

\begin{tabular}{|c|c|c|c|c|c|c|c|c|c|c|c|}
\hline & \multirow[b]{2}{*}{ ROIs } & & \multicolumn{3}{|c|}{ Initially Cued $>$ Absent } & \multicolumn{3}{|c|}{ Initially Uncued $>$ Absent } & \multicolumn{3}{|c|}{ Initially Cued $>$ Initially Uncued } \\
\hline & & & Precue Delay & Cue 1 Delay & Cue 2 Delay & Precue Delay & Cue 1 Delay & Cue 2 Delay & Precue Delay & Cue 1 Delay & Cue 2 Delay \\
\hline \multirow[t]{9}{*}{ Switch Trials } & Contralateral & VOT & $1.4 \times 10^{3}$ & $1.1 \times 10^{8}$ & $1.3 \times 10^{-1}$ & $2.6 \times 10^{-1}$ & $3.7 \times 10^{-2}$ & $1.9 \times 10^{11}$ & $5.5 \times 10^{0}$ & $2.1 \times 10^{10}$ & $1.5 \times 10^{-3}$ \\
\hline & & Parietal & $1.4 \times 10^{0}$ & $5.0 \times 10^{2}$ & $2.2 \times 10^{-2}$ & $2.4 \times 10^{-1}$ & $5.2 \times 10^{-2}$ & $2.4 \times 10^{6}$ & $1.3 \times 10^{-1}$ & $8.8 \times 10^{3}$ & $2.2 \times 10^{-4}$ \\
\hline & & Frontal & $4.1 \times 10^{1}$ & $5.1 \times 10^{1}$ & $5.8 \times 10^{-2}$ & $1.1 \times 10^{1}$ & $2.8 \times 10^{-2}$ & $1.3 \times 10^{1}$ & $5.3 \times 10^{-2}$ & $8.0 \times 10^{1}$ & $4.2 \times 10^{-3}$ \\
\hline & Ipsilateral & VOT & $2.0 \times 10^{-1}$ & $6.4 \times 10^{10}$ & $1.4 \times 10^{-2}$ & $1.4 \times 10^{18}$ & $2.0 \times 10^{8}$ & $1.7 \times 10^{23}$ & $1.5 \times 10^{-4}$ & $4.0 \times 10^{-1}$ & $6.6 \times 10^{-5}$ \\
\hline & & Parietal & $1.5 \times 10^{-1}$ & $1.9 \times 10^{2}$ & $1.8 \times 10^{-2}$ & $6.7 \times 10^{1}$ & $5.9 \times 10^{-2}$ & $8.4 \times 10^{5}$ & $4.5 \times 10^{-3}$ & $1.2 \times 10^{1}$ & $5.1 \times 10^{-3}$ \\
\hline & & Frontal & $4.3 \times 10^{-1}$ & $1.3 \times 10^{2}$ & $3.8 \times 10^{-2}$ & $1.2 \times 10^{0}$ & $5.5 \times 10^{-2}$ & $1.0 \times 10^{1}$ & $1.6 \times 10^{-2}$ & $6.6 \times 10^{3}$ & $1.1 \times 10^{-3}$ \\
\hline & Bilateral & VOT & $1.0 \times 10^{3}$ & $8.4 \times 10^{19}$ & $4.2 \times 10^{-2}$ & $2.0 \times 10^{2}$ & $2.5 \times 10^{4}$ & $5.6 \times 10^{17}$ & $3.4 \times 10^{-2}$ & $1.2 \times 10^{8}$ & $5.2 \times 10^{-5}$ \\
\hline & & Parietal & $2.7 \times 10^{0}$ & $2.1 \times 10^{6}$ & $3.0 \times 10^{-2}$ & $2.6 \times 10^{1}$ & $9.1 \times 10^{-2}$ & $7.7 \times 10^{13}$ & $9.5 \times 10^{-3}$ & $1.1 \times 10^{3}$ & $1.4 \times 10^{-3}$ \\
\hline & & Frontal & $2.9 \times 10^{-1}$ & $1.6 \times 10^{5}$ & $2.3 \times 10^{-2}$ & $6.0 \times 10^{2}$ & $9.0 \times 10^{-2}$ & $9.1 \times 10^{2}$ & $7.2 \times 10^{-3}$ & $3.3 \times 10^{1}$ & $1.5 \times 10^{-3}$ \\
\hline \multirow[t]{9}{*}{ Stay Trials } & Contralateral & VOT & $5.2 \times 10^{0}$ & $3.2 \times 10^{9}$ & $2.0 \times 10^{9}$ & $1.6 \times 10^{1}$ & $2.0 \times 10^{-1}$ & $2.5 \times 10^{-2}$ & $2.4 \times 10^{-2}$ & $1.8 \times 10^{6}$ & $8.7 \times 10^{11}$ \\
\hline & & Parietal & $2.3 \times 10^{-1}$ & $1.3 \times 10^{5}$ & $9.8 \times 10^{32}$ & $1.5 \times 10^{-2}$ & $4.7 \times 10^{-2}$ & $7.9 \times 10^{-2}$ & $3.3 \times 10^{-1}$ & $4.7 \times 10^{3}$ & $3.4 \times 10^{4}$ \\
\hline & & Frontal & $9.5 \times 10^{-3}$ & $4.2 \times 10^{3}$ & $3.8 \times 10^{0}$ & $7.8 \times 10^{-2}$ & $1.2 \times 10^{-1}$ & $7.4 \times 10^{-2}$ & $1.3 \times 10^{-2}$ & $6.96 \times 10^{1}$ & $8.4 \times 10^{-1}$ \\
\hline & Ipsilateral & VOT & $1.3 \times 10^{-1}$ & $3.1 \times 10^{8}$ & $8.3 \times 10^{5}$ & $6.4 \times 10^{21}$ & $8.2 \times 10^{9}$ & $1.6 \times 10^{2}$ & $5.6 \times 10^{-4}$ & $1.4 \times 10^{-1}$ & $1.0 \times 10^{0}$ \\
\hline & & Parietal & $1.3 \times 10^{-1}$ & $2.0 \times 10^{1}$ & $9.3 \times 10^{3}$ & $6.9 \times 10^{-1}$ & $2.5 \times 10^{-1}$ & $2.6 \times 10^{-1}$ & $1.1 \times 10^{-2}$ & $7.6 \times 10^{-1}$ & $3.8 \times 10^{0}$ \\
\hline & & Frontal & $4.3 \times 10^{-2}$ & $1.8 \times 10^{0}$ & $3.2 \times 10^{0}$ & $7.4 \times 10^{-1}$ & $1.2 \times 10^{-1}$ & $3.8 \times 10^{-2}$ & $1.2 \times 10^{-2}$ & $1.2 \times 10^{-1}$ & $3.1 \times 10^{0}$ \\
\hline & Bilateral & VOT & $8.6 \times 10^{-1}$ & $7.4 \times 10^{10}$ & $2.3 \times 10^{11}$ & $2.9 \times 10^{8}$ & $2.9 \times 10^{11}$ & $3.4 \times 10^{0}$ & $1.9 \times 10^{-4}$ & $2.8 \times 10^{4}$ & $8.7 \times 10^{7}$ \\
\hline & & Parietal & $9.4 \times 10^{-1}$ & $8.3 \times 10^{4}$ & $6.0 \times 10^{7}$ & $1.2 \times 10^{1}$ & $1.1 \times 10^{-1}$ & $1.5 \times 10^{-1}$ & $5.9 \times 10^{-2}$ & $5.1 \times 10^{2}$ & $8.8 \times 10^{2}$ \\
\hline & & Frontal & $5.8 \times 10^{-2}$ & $2.7 \times 10^{5}$ & $8.7 \times 10^{2}$ & $1.9 \times 10^{0}$ & $1.0 \times 10^{-1}$ & $1.7 \times 10^{-1}$ & $3.9 \times 10^{-3}$ & $4.8 \times 10^{1}$ & $1.3 \times 10^{1}$ \\
\hline
\end{tabular}

For comparisons associated with Figures $3-6$. All values denoted with scientific notation. 
Table 2. Repeated-measures RANOVA for MVPA Evidences

\begin{tabular}{|c|c|c|c|}
\hline & $D F$, error $D F$ & $F$ & $p$ Value \\
\hline Intercept & 1,9 & 6197.784689 & $4.36 \times 10^{-14}$ \\
\hline ROI & 1,9 & 4001.467168 & $3.11 \times 10^{-13}$ \\
\hline Task & 1,9 & 2.176770995 & 0.174212921 \\
\hline SearchArray & 2,18 & 65.00113086 & $5.82 \times 10^{-09}$ \\
\hline Cue & 2,18 & 56.96416349 & $1.64 \times 10^{-08}$ \\
\hline Hemisphere & 1,9 & 5.238856899 & 0.047864226 \\
\hline ROI:Task & 1,9 & 0.157209716 & 0.700975424 \\
\hline ROI:SearchArray & 2,18 & 5.020507377 & 0.018505849 \\
\hline Task:SearchArray & 2,18 & 1.360941559 & 0.281572067 \\
\hline ROI:Cue & 2,18 & 2.240447544 & 0.135247783 \\
\hline Task:Cue & 2,18 & 20.91164072 & $2.02 \times 10^{-5}$ \\
\hline SearchArray:Cue & 4,36 & 19.26070931 & $1.51 \times 10^{-08}$ \\
\hline ROI:Hemisphere & 1,9 & 1.669684336 & 0.228491381 \\
\hline Task:Hemisphere & 1,9 & 21.23022322 & 0.0012766 \\
\hline SearchArray:Hemisphere & 2,18 & 7.400476712 & 0.004513094 \\
\hline Cue:Hemisphere & 2,18 & 38.09507894 & $3.4 \times 10^{-07}$ \\
\hline ROI:Task:SearchArray & 2,18 & 0.808264561 & 0.461161496 \\
\hline ROI:Task:Cue & 2,18 & 0.580374727 & 0.569823187 \\
\hline ROI:SearchArray:Cue & 4,36 & 0.182197409 & 0.946146413 \\
\hline Task:SearchArray:Cue & 4,36 & 27.32355873 & $1.80 \times 10^{-10}$ \\
\hline ROI:Task:Hemisphere & 1,9 & 21.49003617 & 0.001226657 \\
\hline ROI:SearchArray:Hemisphere & 2,18 & 8.177996188 & 0.00297461 \\
\hline Task:SearchArray:Hemisphere & 2,18 & 8.703949508 & 0.00226752 \\
\hline ROI:Cue:Hemisphere & 2,18 & 13.17584571 & 0.000298718 \\
\hline Task:Cue:Hemisphere & 2,18 & 0.131699021 & 0.877441731 \\
\hline SearchArray:Cue:Hemisphere & 4,36 & 3.335164334 & 0.020144599 \\
\hline ROI:Task:SearchArray:Cue & 4,36 & 1.090391212 & 0.375945056 \\
\hline ROI:Task:SearchArray:Hemisphere & 2,18 & 6.195490136 & 0.008969127 \\
\hline ROI:Task:Cue:Hemisphere & 2,18 & 0.110860231 & 0.895670204 \\
\hline ROI:SearchArray:Cue:Hemisphere & 4,36 & 1.316064038 & 0.282581781 \\
\hline Task:SearchArray:Cue:Hemisphere & 4,36 & 1.701875362 & 0.17098258 \\
\hline ROI:Task:SearchArray:Cue:Hemisphere & 4,36 & 0.908520765 & 0.469378907 \\
\hline
\end{tabular}

Here, "ROI" indicates the comparison between frontal-parietal areas (both coded as the same to effectively average across) versus VOT cortex (see Methods). "Task" indicates stay versus switch trials; "Cue" indicates whether a donut, abacus, or face was cued; "Search Array" indicates which period the data were extracted from (after the first search array or second); and "Hemisphere" indicates whether the data are taken from the hemisphere ipsilateral or contralateral to the cue.

Search Array 1 persisted across Search Array 2 (cued $>$ uncued $\mathrm{BF}=14$; cued $>$ absent $\mathrm{BF}=877$; uncued $>$ absent BF $=0.18$ ). After Switch cues, MVPA evidence for the two stimuli crossed over, with MVPA evidence for the initially unprioritized (but now prioritized) stimulus taking on a value comparable that of the initially cued item during Search Array 1 (now-cued $>$ absent BF $=915$; initially cued in Search Array $1>$ now-cued in search array $2 \mathrm{BF}=$ 0.0310), and MVPA evidence for the initially prioritized (but now unprioritized) stimulus dropping to baseline 
levels for much of Search Array 2 (now uncued $>$ baseline $\mathrm{BF}=0.02)$.

\section{Effects within Unilateral ROIs}

Whereas Figure 3 illustrates the effects of attention on stimulus representations expected to be equated in terms of their bottom-up properties, our design also allowed us to examine the effects of attention in regions in which stimulus representation was expected to be inherently weak, because of the fact that each item in the search array appeared in a separate visual hemifield. Thus, we next assessed the effects of prioritization as a function of whether the cued stimulus appeared contra- or ipsilateral to a given brain region.

In VOT cortex, the effects of selection on the unilateral representation of an item can be summarized as being comparable to the effect of moving it from one hemisphere to the other: In the hemisphere ipsilateral to the cued item, the representation of that item was strengthened to a level comparable to that of the contralateral item. In the hemisphere ipsilateral to the uncued item, in contrast, the representation of that item dropped to baseline levels (Figure 4). When VOT ROIs were broken out by hemisphere, delayperiod stimulus representation before Cue 1 was stronger for the contralateral than the ipsilateral item in three of the four analysis cells, the effect combined across all four strongly favoring greater contra-than-ipsilateral stimulus representation (multiplied $\mathrm{BF}=1.58 \times 10^{6}$ ). With the onset of Cue 1, the effect in the ROI contralateral to the cued item was to trigger precipitous drops in MVPA evidence for both the absent category and the uncued stimulus, the two not differing during Search Array 1 (uncued $>$ absent Stay $\mathrm{BF}=0.2 ;$ Switch $\mathrm{BF}=0.04)$, and the cued stimulus differing from both during Search Array 1 (cued $>$ uncued Stay $\mathrm{BF}=1.83 \times 10^{6}$; cued $>$ absent Stay $\mathrm{BF}=3.20 \times 10^{9}$; cued $>$ uncued Switch BF $=2.16 \times 10^{10}$; cued $>$ absent Switch BF $=1.14 \times 10^{8}$ ). The effect in the ROI ipsilateral to the cue was to trigger a drop in MVPA evidence for the absent category, with MVPA evidence for both the cued item and the uncued item maintained at a level above baseline and comparable to each other (cued $>$ uncued Stay $\mathrm{BF}=0.15$; cued $>$ absent Stay $\mathrm{BF}=3.18 \times 10^{8}$; cued $>$ uncued Switch BF $=0.4$; cued $>$ absent Switch $\mathrm{BF}=6.45 \times 10^{10}$ ).

Now turning to the second search array of the trial, after Stay cues, the pattern from Search Array 1 persisted across Search Array 2 in both hemispheres (for the contralateral hemisphere: cued $>$ uncued $\mathrm{BF}=8.73 \times 10^{11}$; cued $>$ absent $\mathrm{BF}=2.06 \times 10^{9}$; uncued $>$ absent $\mathrm{BF}=0.025$, and for the ipsilateral hemisphere: cued $>$ uncued $\mathrm{BF}=$ 1.08; cued $>$ absent $\mathrm{BF}=8.35 \times 10^{5}$; uncued $>$ absent $\mathrm{BF}=166)$. After Switch cues, in the ROI initially contralateral to the item cued by Cue 1, MVPA evidence for the two stimuli crossed over, with MVPA evidence for the initially uncued stimulus taking on a value comparable to that of the initially cued item (Figure 4A; top right, blue trace; now-cued $>$ uncued $\mathrm{BF}=637$; now-cued $>$ absent $\mathrm{BF}=$ $1.91 \times 10^{11}$; SA1 cued $>$ SA2 cued BF $\left.=7.082\right)$, and the initially cued item now at the level of baseline (Figure 4, top right, red trace; now-uncued $>$ absent $\mathrm{BF}=.14$ ). A crossover was also seen in the ROI initially ipsilateral to the item cued by Cue 1, with the initially uncued stimulus taking on a value comparable to that of the initially cued item (Figure 4B, bottom right, blue trace; now-cued $>$ now-uncued $\mathrm{BF}=1.51 \times 10^{4} ;$ now-cued $>$ absent $\mathrm{BF}=$ $1.72 \times 10^{23}$; SA1 cued $>$ SA2 cued BF $\left.=1.222\right)$, and the initially cued item now at the level of baseline (Figure 4B bottom left, red trace; now-uncued $>$ absent $\mathrm{BF}=0.015)$.

In parietal and in frontal cortex, the effects of attention within unilateral ROIs were not qualitatively different from what they had been in bilateral ROIs (see Table 1 for BFs).
Figure 5. Time course of classifier evidence in the two-item task, in unilateral parietal ROIs. All graphical conventions are the same as Figure 3 .

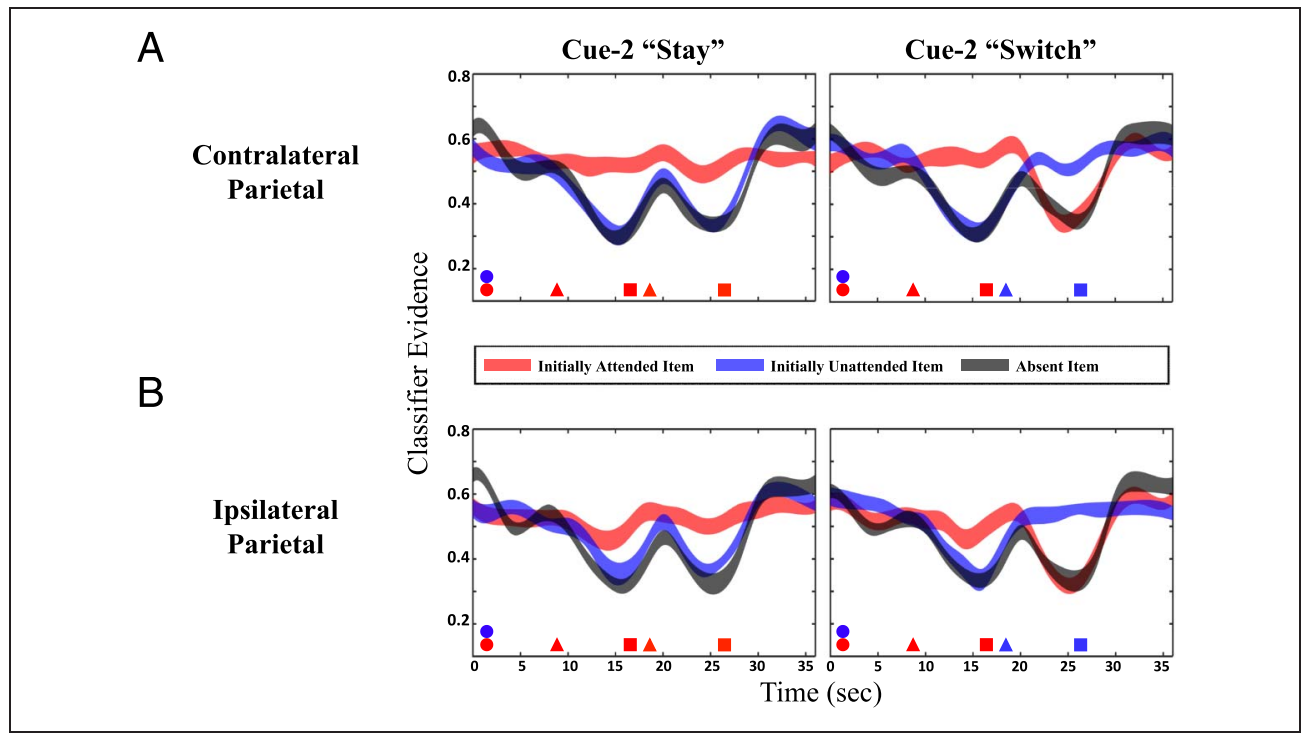


Figure 6. Time course of classifier evidence in the two-item task, in unilateral frontal ROIs. All graphical conventions are the same as Figure 3.

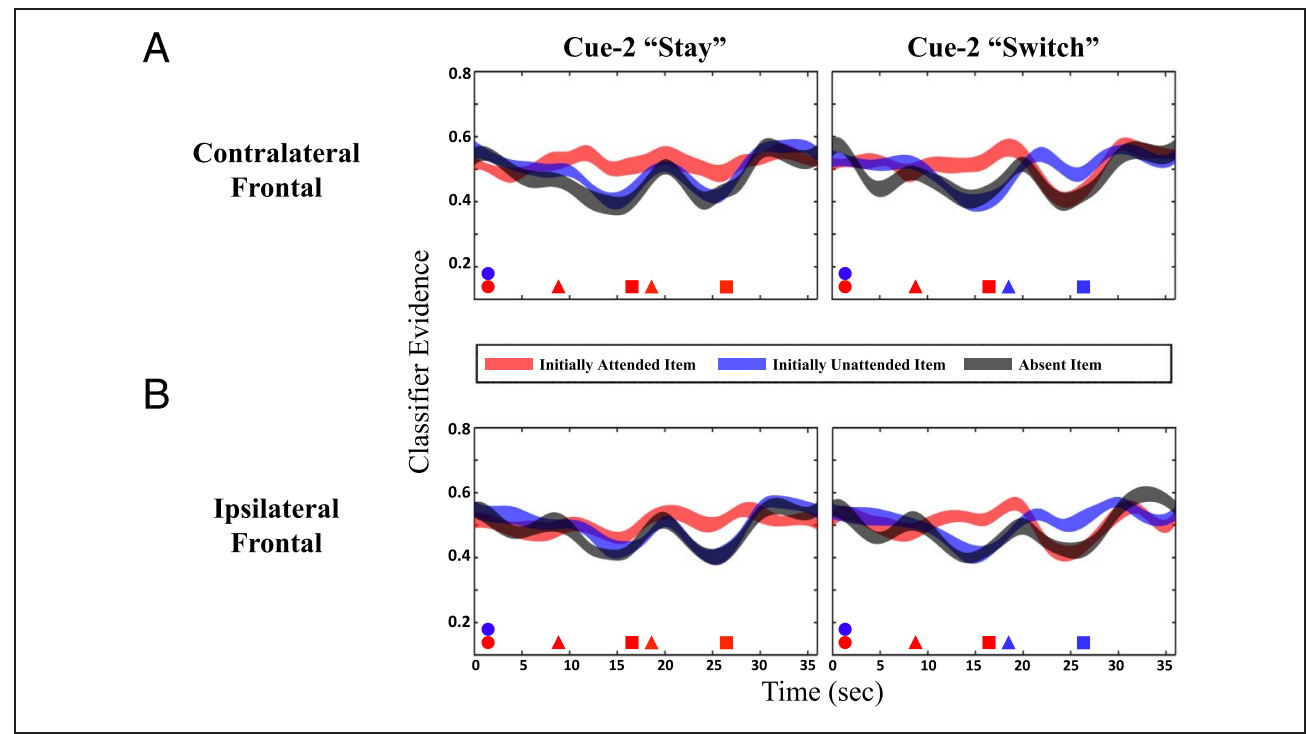

\section{Comparison between VOT Cortex and}

\section{Frontoparietal Cortex}

To quantitatively compare the effects of attentional cuing on stimulus information in the VOT versus frontoparietal ROIs, we ran linear mixed effects models and computed two repeated-measures ANOVAs, one on search array data from bilateral ROIs and one on search array data broken out by unilateral ROIs (see Methods). The complete sets of effects are reported in Table 2, and here, we restrict the reporting to the most theoretically consequential contrasts. Although visual inspection of Figure 3 suggests that these two regions may differ in that only in the bilateral VOT ROI was the uncued stimulus represented at abovebaseline levels, the ANOVA on data from the bilateral ROIs revealed no Cue $\times$ ROI interaction, $F(2,36)=2.24$; $p=.135$. The difference between the two ROIs was confirmed, however, when the data were broken out by hemisphere (Figures 4-6), in the form of a significant three-way interaction of Cue $\times$ Hemisphere (contralateral, ipsilateral) $\times$ ROI, $F(2,36)=13.176 ; p=.000299$, averaging across trial type (i.e., stay vs. switch) and search array

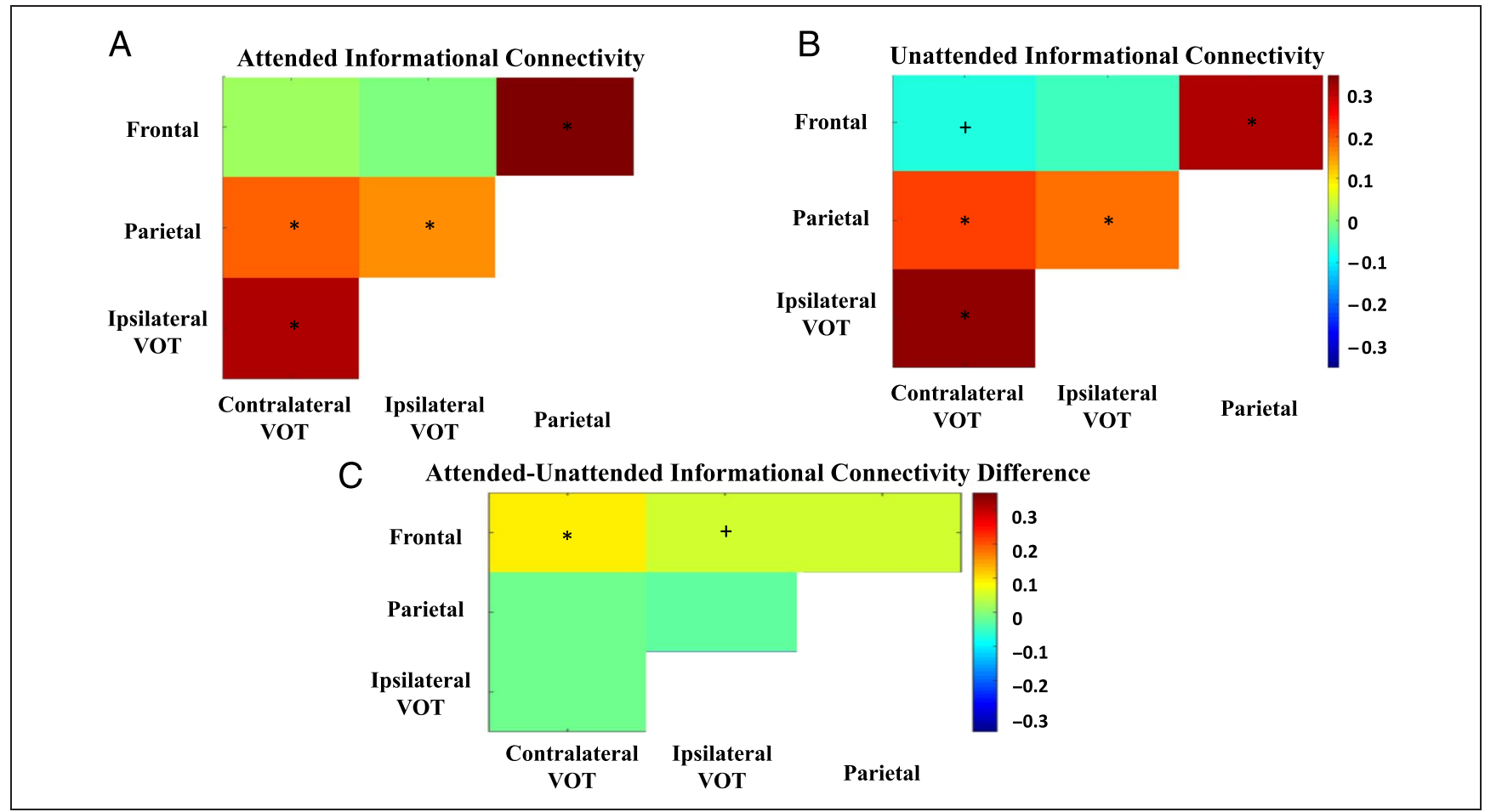

Figure 7. IC. IC between ROIs for the item that was cued (A) and, therefore, presumed to have been attended or uncued (B), and, therefore, presumed to have not been attended. $* p$ corrected $<.05 ;+p<.05$ uncorrected. The difference between the attended and unattended ICs is plotted in C. For full list of values, see Table 3. 
period. This confirms the statistical reliability of the difference evident from comparing Figure 4 with Figures 5 and 6: Only in VOT cortex, in the hemisphere ipsilateral to the cued visual field, did attention have the effect of boosting the representation of the cued item and weakening the representation of the uncued item. Importantly, evidence for a difference between the ROIs disappears when cue information is not taken into account (two-way interaction of ROI $\times$ Hemisphere: $F(1,36)=1.67 ; p=0.228)$, indicating that the critical three-way interaction is not merely driven by a baseline shift in all evidences between regions as a function of laterality (Figure 6).

\section{Effects of Attentional Prioritization on IC between Brain Regions}

Because the preceding analyses indicated that parietal and frontal regions maintained an active representation of only the attended stimulus, despite the highly salient presence on the screen of two stimuli, we speculated that these representations might have a functional role in implementing top-down attentional control. Importantly, previous work has established that fluctuations in signal intensity in frontal and parietal regions during a selective attention task correlates with MVPA decoding performance in VOT cortex (Nelissen et al., 2013). Here, we extended this approach by asking the more specific question of whether the parietal and/or frontal representations of category information that we observed in this study might, themselves, have had a role in the control of attentional selection. We did so by assessing the strength of correlation, over time, of MVPA decoding performance between regions, a measure known as IC (Coutanche \& Thompson-Schill, 2014). The results indicated that, although IC between several regions was elevated during the task, relative to baseline (Figure 7; see Table 3 for values), the specific patterns of IC with VOT differed between the frontal and the parietal ROIs, in that only for the frontal-to-contralateral VOT

Table 3. IC Values and $p$ Values

\begin{tabular}{|c|c|c|c|}
\hline ROI Pair & IC Value & p Value (Uncorrected) & Bayes Factor \\
\hline \multicolumn{4}{|l|}{ Attended } \\
\hline Frontal<->Contralateral VOT & 0.0146 & $1.7515(0.3503)$ & 0.0517 \\
\hline Frontal<-> Ipsilateral VOT & -0.0028 & $2.156(0.539)$ & 0.0495 \\
\hline Frontal $<->$ Parietal & 0.3616 & $0(0)$ & $2.6892 \times 10^{9}$ \\
\hline Parietal $<->$ Contralateral VOT & 0.1883 & $0(0)$ & 6.5636 \\
\hline Parietal<-> Ipsilateral VOT & 0.156 & $0(0)$ & 15.5251 \\
\hline Ipsilateral VOT $<->$ Contralateral VOT & 0.3152 & $0(0)$ & 1216.5216 \\
\hline \multicolumn{4}{|l|}{ Unattended } \\
\hline Frontal $<->$ Contralateral VOT & -0.0757 & $0.2688(0.0336)$ & 0.2805 \\
\hline Frontal $<->$ Ipsilateral VOT & -0.0473 & $0.4025(0.0575)$ & 0.1257 \\
\hline Frontal<-> Parietal & 0.315 & $0(0)$ & $2.6181 \times 10^{4}$ \\
\hline Parietal<->Contralateral VOT & 0.2088 & $0.01(0.0001)$ & 8.4791 \\
\hline Parietal<-> Ipsilateral VOT & 0.18097 & $0.01(0.0001)$ & 4.2689 \\
\hline Ipsilateral VOT $<->$ Contralateral VOT & 0.3317 & $0(0)$ & $7.4521 \times 10^{7}$ \\
\hline \multicolumn{4}{|l|}{ Attended-Unattended Difference } \\
\hline Frontal $<->$ Contralateral VOT & 0.0904 & $0(0)$ & 5.74 \\
\hline Frontal<-> Ipsilateral VOT & 0.0444 & $0.0864(0.0096)$ & 0.3525 \\
\hline Frontal $<->$ Parietal & 0.0464 & $0.6138(0.1023)$ & 0.0993 \\
\hline Parietal<->Contralateral VOT & -0.0206 & $1.3858(0.6929)$ & 0.0595 \\
\hline Parietal<-> Ipsilateral VOT & -0.0249 & $0.774(0.774)$ & 0.0568 \\
\hline Ipsilateral VOT<->Contralateral VOT & -0.0165 & $2.0127(0.6709)$ & 0.0521 \\
\hline
\end{tabular}

All values denoted with scientific notation. $p$ Values were computed by bootstrapping procedure (see Methods). Bayes factors calculated via same method as in Figures 3-6 (see also Methods). 
connection did it vary as a function of the attentional status of a stimulus within a trial. More specifically, whereas IC for the cued item between the (bilateral) frontal ROI and the unilateral VOT ROI contralateral to the attended item was near 0 ( $\mathrm{IC}=0.0146 ; p=0.364 ; \mathrm{BF}=0.0517)$, IC between these regions for the uncued item was numerically negative (uncorrected IC $=-0.076, p=.037, p$ corrected $=$ $0.279 ; \mathrm{BF}=0.2805)$, such that these IC differed significantly from each other $(\mathrm{IC}$ diff $=0.09 ; p($ corrected $)<$ $0.0001 ; \mathrm{BF}=5.74)$. This pattern is consistent with the possibility that the frontal cortex may actively control, via an inhibitory influence, the strength of the representation of the uncued item.

\section{DISCUSSION}

Biased competition and, more recently, divisive normalization (Carandini \& Heeger, 2011), are principles that have been enormously influential on our understanding of the neural bases of attention. At the level of single neurons in visual cortex, attention to one of two simultaneously presented stimuli has the effect of resolving the competition that is inferred by comparing the response to this pair of stimuli to the response to the attended stimulus alone (Desimone, 1998). More generally, attention is hypothesized to bias the competition for representation of whole objects and, therefore, to operate at multiple levels of distributed population-level representations (Valdés-Sosa, Iglesias-Fuster, \& Torres, 2014). We used multivariate analysis methods to quantify the temporally dynamic effects of selective attention on population-level neural representations of visually presented stimuli in a change-detection task. We observed that attention selectively enhanced the representational fidelity of the attended stimulus in both hemispheres of visual cortex, whether contra- or ipsilateral to the cued item, and that it did so at the expense of the unattended stimulus. This effect was most pronounced in the hemisphere contralateral to the attended stimulus, where the unattended representation became indistinguishable from baseline, and unfolded slowly over 4-6 sec, mirroring the time course observed in tasks of working memory requiring analogous attentional shifts between memoranda (Lewis-Peacock, Drysdale, Oberauer, \& Postle, 2012). In contrast, parietal and frontal cortex showed abovebaseline evidence of stimulus representation only for the attended item, regardless of hemifield in which it was presented (with shifts of attention manifesting over a similar time course). To some extent, this difference in laterality might be viewed as simply a consequence of the general trend that stimulus representations become more abstract and more spatially invariant as one moves rostrally in the brain. On the other hand, this difference might also reflect a fundamental difference in the functional role of these different regions in visual-selective attention.

For decades, a widely held view has been that one can construe the visual attentional functions of frontoparietal cortex as acting as a source of top-down attentional control, and of VOT cortex as a site of its actions. This functional distinction has recently been called into question after a multivariate analysis of fMRI data showed that stimulus representations in VOT, parietal, and frontal cortex all demonstrated sensitivity to manipulations of attention (Ester et al., 2016). An important limitation of that study, however, was that the authors only tracked the neural representation of one item per trial, and were thus unable to examine the effects within a region when attention was withdrawn from one stimulus and allocated to another. If one were to consider, for example, the frontal representation of only the initially cued item during switch trials of our change detection task (Figure 3C), one would observe that this representation transitioned from intermediate strength during the initial delay (before selection), to elevated strength after being cued, to drastically lower strength after attention was reallocated to a different stimulus. What one would not see, however, is that the mirror-opposite time course of the initially unattended item suggests an all-or-none pattern of attentionally sensitive representations in frontal cortex, and that this differs qualitatively from the more modulatory effects of attention observed in VOT cortex.

Although many previous studies have shown a correlation of activity between putatively control-related and stimulus-representing regions during selective attention tasks (Baldauf \& Desimone, 2014; Gazzaley, Cooney, McEvoy, Knight, \& D'Esposito, 2005), including the covariation of frontoparietal signal intensity with VOT MVPA performance (Nelissen et al., 2013), a novel aspect of this experiment is the evaluation of IC between these regions. This approach, unlike functional connectivity, can identify situations in which two areas are sharing information of interest, as opposed to cases in which the pattern of correlated activity is because of a more general factor, such as fluctuations in motivation or arousal. Indeed, in our study, the IC analysis generated two novel insights. The first is that parietal and frontal functions may differ in that the IC of the former, but not the latter, is invariant to withintrial changes of attentional priority. This invariance of parietal-VOT IC might be expected of the operation of a priority map that tracks all potentially relevant stimuli in the environment regardless of immediate priority status. The second is the manner in which frontal cortex exerts attentional control. The negative-trending IC between frontal cortex and VOT in the hemisphere contralateral to the uncued stimulus would be consistent with a role for the PFC in the active suppression of the neural representation of that item. This raises the tantalizing possibility that one mechanism for biasing the competition for representation among items in a visual scene is to weaken the representation of unprioritized information.

Interpretation of the difference in frontal-VOT IC for the uncued versus for the cued item is complicated by the fact that the statistical significance of the negative-trending IC for the uncued item did not survive correction for multiple comparisons, and the Bayes factor for this comparison 
favored the likelihood that it did not differ from 0 . Thus, the possibility that top-down inhibition from frontal cortex may contribute to the attentional biasing of competition for visual representation is only speculative at this point. (We do note that one possible explanation for the divergence between the inference supported by bootstrapping versus the Bayesian analysis is that, unlike the case for the Bayesian analyses of MVPA evidences, the prior distribution for the IC contrasts was relatively undefined for the theoretical distribution. We therefore selected a prior that made the least number of assumptions, and extended uniformly from -1 to 1 . This increased the likelihood of a Bayes factor that would favor the [more readily defined] null hypothesis in a domain where noise was high and effect size small, as is the case for the IC data.)

Although the general observation that frontal and parietal cortex support at least partly dissociable functions in the control of attention is not surprising, the empirical patterns of IC between frontal, parietal, and VOT ROIs that we observed here are not so straightforward. Why should it be that frontal-VOT IC is near 0 for the attended item, whereas frontal-parietal IC for the attended item and parietal-VOT IC for the attended item are both strongly positive? Quantitatively, this pattern suggests that different components of variance in MVPA evidence in the frontal ROI dominate this region's IC with the VOT versus its IC with the parietal ROI. Mechanistically, this might come about if a principal function for frontal circuits is to represent the current rule, whereas a principle function of parietal cortex is to maintain a priority map. If the rule during a search array is "attend the abacus and ignore the face," the frontal representation of this rule could be expected to be constant and stable across the duration of the trial epoch, and the independence of this rule to moment-to-moment fluctuations of sensory information about the abacus would account for the statistical independence from MVPA evidence in VOT. Because parietal circuits also encode this rule, parietal-frontal IC should be high. In addition, however, because parietal circuits are also maintaining a priority map, they must track the stimuli that are currently flashing on the screen, and will thus be sensitive to fluctuations in the sensory strength of both, resulting in strong and positive parietal-VOT IC for both the cued (attended) and the uncued (unattended) item. Another factor that may influence this pattern in the IC results is the possibility that decoding performance may be inherently different in frontal cortex relative to other brain areas (Bhandari, Gagne, \& Badre, 2018).

The present data provide a benchmark for the effects of selective attention on multivariate estimates of the population-level representation of each of two stimulione prioritized and one unprioritized — when both remain visible and highly salient. An important goal for future research will be to assess the extent to which similar mechanisms may account for the effects of prioritization within the contents of working memory (e.g., LaRocque et al., 2017; Rose et al., 2016; Emrich et al., 2013; Lewis-Peacock et al., 2012). In so doing, it will be important to keep in mind fundamental differences in task demands. For example, one recent study has reported evidence that multivariate evidence for unprioritized information in working memory can be found in IPS and frontal cortex (Christophel et al., 2018). Although this might appear to be at odds with the results reported here, an important difference between the two tasks is that, in this study, the initially uncued item did not need to be retained in memory for the simple reason that it would also be visible as part of Search Array 2. An important question for future research is whether frontal and parietal representations of unprioritized stimulus information are better construed as supporting representation- or control-related functions.

\section{Acknowledgments}

We would like to thank Jason Samaha, Olivia Gosseries, and Marc Coutanche for helpful discussions.

Reprint requests should be sent to Andrew D. Sheldon, Neuroscience Training Program, University of Wisconsin-Madison, Madison, WI 53706, USA, or via e-mail: andrew.sheldon255@ gmail.com.

\section{Author Contributions}

Andrew D. Sheldon: Conceptualization; Data curation; Formal analysis; Investigation; Methodology; Project administration; Writing - original draft; Writing - review \& editing. Elyana Saad: Investigation; Project administration; Writing original draft. Muhammet I. Sahan: Conceptualization. Emma E. Meyering: Data curation; Investigation. Michael J. Starrett: Conceptualization; Data curation. Joshua J. LaRocque: Conceptualization. Nathan S. Rose: Conceptualization. Bradley R. Postle: Conceptualization; Funding acquisition; Resources; Supervision; Writing - review \& editing.

\section{Funding Information}

Andrew D. Sheldon, National Institutes of Health (http://dx .doi.org/10.13039/100000002), grants: T32GM008692 and T32GM750738. Bradley R. Postle, National Institutes of Health grant R01 MH064498.

\section{Diversity in Citation Practices}

A retrospective analysis of the citations in every article published in this journal from 2010 to 2020 has revealed a persistent pattern of gender imbalance: Although the proportions of authorship teams (categorized by estimated gender identification of first author/last author) publishing in the Journal of Cognitive Neuroscience (JoCN) during this period were $\mathrm{M}(\mathrm{an}) / \mathrm{M}=.408, \mathrm{~W}$ (oman) $/ \mathrm{M}=.335$, $\mathrm{M} / \mathrm{W}=.108$, and $\mathrm{W} / \mathrm{W}=.149$, the comparable proportions for the articles that these authorship teams cited were $\mathrm{M} / \mathrm{M}=.579, \mathrm{~W} / \mathrm{M}=.243, \mathrm{M} / \mathrm{W}=.102$, and $\mathrm{W} / \mathrm{W}=.076$ 
(Fulvio et al., JoCN, 33:1, pp. 3-7). Consequently, JoCN encourages all authors to consider gender balance explicitly when selecting which articles to cite and gives them the opportunity to report their article's gender citation balance.

\section{REFERENCES}

Baldauf, D., \& Desimone, R. (2014). Neural mechanisms of object-based attention. Science, 344, 424-427. DOI: https:// doi.org/10.1126/science.1247003, PMID: 24763592

Bettencourt, K. C., \& Xu, Y. (2016). Decoding the content of visual short-term memory under distraction in occipital and parietal areas. Nature Neuroscience, 19, 150-157. DOI: https://doi.org/10.1038/nn.4174, PMID: 26595654, PMCID: PMC4696876

Bhandari, A., Gagne, C., \& Badre, D. (2018). Just above chance: Is it harder to decode information from prefrontal cortex hemodynamic activity patterns? Journal of Cognitive Neuroscience, 30, 1473-1498. DOI: https://doi.org/10.1162 /jocn_a_01291, PMID: 29877764

Bichot, N. P., Heard, M. T., DeGennaro, E. M., \& Desimone, R. (2015). A source for feature-based attention in the prefrontal cortex. Neuron, 88, 832-844. DOI: https://doi.org/10.1016 /j.neuron.2015.10.001, PMID: 26526392, PMCID: PMC4655197

Brady, T. F., Konkle, T., Alvarez, G. A., \& Oliva, A. (2008). Visual long-term memory has a massive storage capacity for object details. Proceedings of the National Academy of Sciences, U.S.A., 105, 14325-14329. DOI: https://doi.org/10.1073/pnas 0803390105, PMID: 18787113, PMCID: PMC2533687

Cai, Y., Sheldon, A. D., Yu, Q., \& Postle, B. R. (2019). Overlapping and distinct contributions of stimulus location and of spatial context to nonspatial visual short-term memory. Journal of Neurophysiology, 121, 1222-1231. DOI: https:// doi.org/10.1152/jn.00062.2019, PMID: 30856041, PMCID: PMC6485733

Carandini, M., \& Heeger, D. J. (2011). Normalization as a canonical neural computation. Nature Reviews Neuroscience, 13, 51-62. DOI: https://doi.org/10.1038/nrn3136, PMID: 22108672, PMCID: PMC3273486

Carlisle, N. B., Arita, J. T., Pardo, D., \& Woodman, G. F. (2011). Attentional templates in visual working memory. Journal of Neuroscience, 31, 9315-9322. DOI: https://doi.org/10.1523 /JNEUROSCI.1097-11.2011, PMID: 21697381, PMCID: PMC3147306

Christophel, T. B., \& Haynes, J.-D. (2014). Decoding complex flow-field patterns in visual working memory. Neuroimage, 91, 43-51. DOI: https://doi.org/10.1016/j.neuroimage.2014 .01.025, PMID: 24480302

Christophel, T. B., Hebart, M. N., \& Haynes, J.-D. (2012). Decoding the contents of visual short-term memory from human visual and parietal cortex. Journal of Neuroscience, 32, 12983-12989. DOI: https://doi.org/10.1523/JNEUROSCI .0184-12.2012, PMID: 22993415, PMCID: PMC6621473 Christophel, T. B., Iamshchinina, P., Yan, C., Allefeld, C., \& Haynes, J.-D. (2018). Cortical specialization for attended versus unattended working memory. Nature Neuroscience, 21, 494-496. DOI: https://doi.org/10.1038/s41593-018-0094-4, PMID: 29507410

Christophel, T. B., Klink, P. C., Spitzer, B., Roelfsema, P. R., \& Haynes, J.-D. (2017). The distributed nature of working memory. Trends in Cognitive Sciences, 21, 111-124. DOI: https://doi.org/10.1016/j.tics.2016.12.007, PMID: 28063661

Cohen, E. H., \& Tong, F. (2015). Neural mechanisms of objectbased attention. Cerebral Cortex, 25, 1080-1092. DOI: https://doi.org/10.1093/cercor/bht303, PMID: 24217991, PMCID: PMC4380003
Corbetta, M., \& Shulman, G. L. (2002). Control of goal-directed and stimulus-driven attention in the brain. Nature Reviews Neuroscience, 3, 201-215. DOI: https://doi.org/10.1038/nrn755, PMID: 11994752

Coutanche, M. N., \& Thompson-Schill, S. L. (2014). Using informational connectivity to measure the synchronous emergence of fMRI multi-voxel information across time. Journal of Visualized Experiments, 51226. DOI: https://doi .org/10.3791/51226, PMID: 25046335, PMCID: PMC4210113

Desimone, R. (1998). Visual attention mediated by biased competition in extrastriate visual cortex. Philosophical Transactions of the Royal Society of London, Series B: Biological Sciences, 353, 1245-1255. DOI: https://doi.org/10.1098/rstb 1998.0280, PMID: 9770219, PMCID: PMC1692333

Desimone, R., \& Duncan, J. (1995). Neural mechanisms of selective visual attention. Annual Review of Neuroscience, 18, 193-222. DOI: https://doi.org/10.1146/annurev.ne.18 .030195.001205, PMID: 7605061

Dienes, Z. (2014). Using Bayes to get the most out of nonsignificant results. Frontiers in Psychology, 5, 781. DOI: https://doi.org/10.3389/fpsyg.2014.00781, PMID: 25120503, PMCID: PMC 4114196

Emrich, S. M., Riggall, A. C., Larocque, J. J., \& Postle, B. R. (2013). Distributed patterns of activity in sensory cortex reflect the precision of multiple items maintained in visual short-term memory. Journal of Neuroscience, 33, 6516-6523. DOI: https://doi.org/10.1523/JNEUROSCI.5732-12.2013, PMID: 23575849, PMCID: PMC3664518

Ester, E. F., Sutterer, D. W., Serences, J. T., \& Awh, E. (2016). Feature-selective attentional modulations in human frontoparietal cortex. Journal of Neuroscience, 36, 8188-8199. DOI: https://doi.org/10.1523/JNEUROSCI.3935-15.2016, PMID: 27488638, PMCID: PMC4971365

Gazzaley, A., Cooney, J. W., McEvoy, K., Knight, R. T., \& D'Esposito, M. (2005). Top-down enhancement and suppression of the magnitude and speed of neural activity. Journal of Cognitive Neuroscience, 17, 507-517. DOI: https://doi.org/10.1162/0898929053279522, PMID: 15814009

Gosseries, O., Yu, Q., LaRocque, J. J., Starrett, M. J., Rose, N. S., Cowan, N., et al. (2018). Parietal-occipital interactions underlying control- and representation-related processes in working memory for nonspatial visual features. Journal of Neuroscience, 38, 4357-4366. DOI: https://doi.org/10.1523 /JNEUROSCI.2747-17.2018, PMID: 29636395, PMCID: PMC5932644

Hamker, F. H. (2005). The reentry hypothesis: The putative interaction of the frontal eye field, ventrolateral prefrontal cortex, and areas V4, IT for attention and eye movement. Cerebral Cortex, 15, 431-447. DOI: https://doi.org/10.1093 /cercor/bhh146, PMID: 15749987

Haxby, J. V., Gobbini, M. I., Furey, M. L., Ishai, A., Schouten, J. L., \& Pietrini, P. (2001). Distributed and overlapping representations of faces and objects in ventral temporal cortex. Science, 293, 2425-2430. DOI: https://doi.org/10.1126/science.1063736, PMID: 11577229

Huth, A. G., Lee, T., Nishimoto, S., Bilenko, N. Y., Vu, A. T., \& Gallant, J. L. (2016). Decoding the semantic content of natural movies from human brain activity. Frontiers in Systems Neuroscience, 10, 81. DOI: https://doi.org/10.3389 /fnsys.2016.00081, PMID: 27781035, PMCID: PMC5057448

LaRocque, J. J., Riggall, A. C., Emrich, S. M., \& Postle, B. R. (2017). Within-category decoding of information in different attentional states in short-term memory. Cerebral Cortex, 27, 4881-4890. DOI: https://doi.org/10.1093/cercor/bhw283, PMID: 27702811, PMCID: PMC6059111

Lewis-Peacock, J. A., Drysdale, A. T., Oberauer, K., \& Postle, B. R. (2012). Neural evidence for a distinction between short-term memory and the focus of attention. Journal of 
Cognitive Neuroscience, 24, 61-79. DOI: https://doi.org/10. 1162/jocn a 00140, PMID: 21955164, PMCID: PMC3222712

Moore, T., \& Armstrong, K. M. (2003). Selective gating of visual signals by microstimulation of frontal cortex. Nature, 421, 370-373. DOI: https://doi.org/10.1038/nature01341, PMID: 12540901

Moore, T., \& Zirnsak, M. (2017). Neural mechanisms of selective visual attention. Annual Review of Psychology, 68, 47-72.

DOI: https://doi.org/10.1146/annurev-psych-122414-033400, PMID: 28051934

Nelissen, N., Stokes, M., Nobre, A. C., \& Rushworth, M. F. S. (2013). Frontal and parietal cortical interactions with distributed visual representations during selective attention and action selection. Journal of Neuroscience, 33, 16443-16458. DOI: https://doi.org/10.1523/JNEUROSCI.2625-13.2013, PMID: 24133250, PMCID: PMC3797369

Reddy, L., Kanwisher, N. G., \& VanRullen, R. (2009). Attention and biased competition in multi-voxel object representations Proceedings of the National Academy of Sciences, U.S.A., 106, 21447-21452. DOI: https://doi.org/10.1073/pnas .0907330106, PMID: 19955434, PMCID: PMC2795499

Reinhart, R. M. G., \& Woodman, G. F. (2014). High stakes trigger the use of multiple memories to enhance the control of attention. Cerebral Cortex, 24, 2022-2035. DOI: https://doi.org/10.1093/cercor/bht057, PMID: 23448876, PMCID: PMC 4089381

Rose, N. S., LaRocque, J. J., Riggall, A. C., Gosseries, O., Starrett, M. J., Meyering, E. E., et al. (2016). Reactivation of latent working memories with transcranial magnetic stimulation. Science, 354, 1136-1139. DOI: https://doi.org/10.1126 /science.aah7011, PMID: 27934762, PMCID: PMC5221753

Sprague, T. C., Saproo, S., \& Serences, J. T. (2015). Visual attention mitigates information loss in small- and large-scale neural codes. Trends in Cognitive Sciences, 19, 215-226. DOI: https://doi.org/10.1016/j.tics.2015.02.005, PMID: 25769502, PMCID: PMC4532299

Valdés-Sosa, M. J., Iglesias-Fuster, J., \& Torres, R. (2014). Attentional selection of levels within hierarchically organized figures is mediated by object-files. Frontiers in Integrative Neuroscience, 8, 91. DOI: https://doi.org/10.3389/fnint.2014 .00091, PMID: 25565994, PMCID: PMC4267176

van Bergen, R. S., Ma, W. J., Pratte, M. S., \& Jehee, J. F. M. (2015). Sensory uncertainty decoded from visual cortex predicts behavior. Nature Neuroscience, 18, 1728-1730. DOI: https://doi.org/10.1038/nn.4150, PMID: 26502262, PMCID: PMC 4670781

Van der Stigchel, S. V., \& Olivers, C. N. L. (2019). The flexible nature of the interaction between attention and working memory. Journal of Cognition, 2, 31. DOI: https://doi .org/10.5334/joc.68, PMID: 31517242, PMCID: PMC6688549

Zhang, Y., Meyers, E. M., Bichot, N. P., Serre, T., Poggio, T. A., \& Desimone, R. (2011). Object decoding with attention in inferior temporal cortex. Proceedings of the National Academy of Sciences, U.S.A., 108, 8850-8855. DOI: https:// doi.org/10.1073/pnas.1100999108, PMID: 21555594, PMCID: PMC3102370 